\title{
Terminkalender 2019
}

https://doi.org/10.1515/iwp-2019-0017

\begin{tabular}{|c|c|c|}
\hline $\begin{array}{l}\text { 4. bis 8. März } \\
\text { Frankfurt am Main }\end{array}$ & DGI-Zertifikatslehrgang Informationsassistenz & $\begin{array}{l}\text { DGI-Geschäftsstelle, Windmühlstraße 3, } 60329 \text { Frankfurt am } \\
\text { Main, Telefon } 069 \text { 430313, Fax } 069 \text { 4909096, } \\
\text { informationsassistent@dgi-info.de, www.dgi-info.de }\end{array}$ \\
\hline $\begin{array}{l}\text { 5. bis 6. März } \\
\text { Düsseldorf }\end{array}$ & $\begin{array}{l}\text { 15. DVMD-Fachtagung } \\
\text { Medizinisches Informationsmanagement } \\
\text { Brücken bauen zwischen Medizin, Informatik, } \\
\text { Biometrie und Epidemiologie }\end{array}$ & $\begin{array}{l}\text { DVMD-Geschäftsstelle, Lobdengaustraße 13, } 69493 \\
\text { Hirschberg, Telefon 06201 4891884, dvmd@dvmd.de, } \\
\text { www.dvmd.de, www.dvmd-tagung.de }\end{array}$ \\
\hline $\begin{array}{l}\text { 7. März } \\
\text { Frankfurt am Main }\end{array}$ & DGI-Praxisseminar Teambildung mit Metadaten & $\begin{array}{l}\text { DGI-Geschäftsstelle, Windmühlstraße 3, } 60329 \text { Frankfurt am } \\
\text { Main, Telefon } 069430313 \text {, Fax } 069 \text { 4909096, } \\
\text { informationsassistent@dgi-info.de, } \\
\text { www.dgi-info.de }\end{array}$ \\
\hline $\begin{array}{l}\text { 7. bis 8. März } \\
\text { Mannheim }\end{array}$ & $\begin{array}{l}6^{\text {th }} \text { European User Conference } \\
\text { Microdata from Eurostat }\end{array}$ & $\begin{array}{l}\text { Heike Wirth und Oshrat Hochman, GESIS-Leibniz-Institut für } \\
\text { Sozialwissenschaften Mannheim, B2,1, 68159 Mannheim, } \\
\text { heike.wirth@gesis.org, oshrat.hochman@gesis.org, } \\
\text { https://www.gesis.org/en/services/events/gesis-conferen } \\
\text { ces/european-user-conference-6/ }\end{array}$ \\
\hline $\begin{array}{l}\text { 11. bis 15. März } \\
\text { Frankfurt am Main }\end{array}$ & DGI-Zertifikatslehrgang Informationsassistenz & $\begin{array}{l}\text { DGI-Geschäftsstelle, Windmühlstraße 3, } 60329 \text { Frankfurt am } \\
\text { Main, Telefon } 069 \text { 430313, Fax } 069 \text { 4909096, } \\
\text { informationsassistent@dgi-info.de, www.dgi-info.de }\end{array}$ \\
\hline $\begin{array}{l}\text { 14. März } \\
\text { München }\end{array}$ & DIGITALQUADRAT & $\begin{array}{l}\text { Marketing Projekt } 2000 \mathrm{GmbH} \text {, Birgit Reber, Hochstraße 3, } \\
86453 \text { Dasing, Telefon } 08205 \text { 96233, birgit.- } \\
\text { reber@digitusmagazin.de, https://digital-quadrat.de }\end{array}$ \\
\hline $\begin{array}{l}\text { 18. bis 20. März } \\
\text { Potsdam }\end{array}$ & $\begin{array}{l}\text { 10. Konferenz Professionelles } \\
\text { Wissensmanagement: Wissensmanagement in } \\
\text { digitalen Arbeitswelten - Aktuelle Ansätze und } \\
\text { Perspektiven }\end{array}$ & $\begin{array}{l}\text { Prof. Dr.-Ing. Peter Heisig, Fachbereich } \\
\text { Informationswissenschaften, Fachhochschule Potsdam, } \\
\text { Kiepenheuerallee 5, } 14469 \text { Potsdam, Telefon 0331580-1520, } \\
\text { heisig@fh-potsdam.de }\end{array}$ \\
\hline $\begin{array}{l}\text { 18. bis 21. März } \\
\text { Leipzig }\end{array}$ & $\begin{array}{l}\text { 7. Bibliothekskongress Leipzig } 2019 \\
\text { 108. Deutscher Bibliothekartag } \\
\text { Bibliotheken verändern } \\
\text { Gastland Niederlande }\end{array}$ & $\begin{array}{l}\text { BID-Geschäftsstelle, c/o Deutscher Bibliotheksverband, } \\
\text { Fritschestraße 27-28, } 10585 \text { Berlin, Telefon } 03 \text { 6449899-0, } \\
\text { Fax } 030 \text { 6449899-27, bid@bideutschland.de, } \\
\text { www.bideutschland.de }\end{array}$ \\
\hline $\begin{array}{l}\text { 19. bis 20. März } \\
\text { Berlin }\end{array}$ & Open Science Conference 2019 & $\begin{array}{l}\text { Melanie Lorenzen und Dr. Guido Scherp, Leibniz- } \\
\text { Forschungsverbund Science 2.0, osc@zbw.eu, } \\
\text { g.scherp@zbw.eu, www.open-science-conference.eu/ }\end{array}$ \\
\hline $\begin{array}{l}\text { 21. März } \\
\text { Frankfurt am Main }\end{array}$ & dcif-Impulsveranstaltung & $\begin{array}{l}\text { dcif e.V. - Bundesverband Wettbewerbs- und Marktanalyse, } \\
\text { Morsbach } 72 \text { a, } 42857 \text { Remscheid, Telefon } 219169232 \text { 42, } \\
\text { info@dcif.de }\end{array}$ \\
\hline $\begin{array}{l}\text { 21. bis 24. März } \\
\text { Leipzig }\end{array}$ & Leipziger Buchmesse & $\begin{array}{l}\text { Leipziger Messe GmbH, Projektteam Buchmesse, Messe-Allee } \\
\text { 1, } 04356 \text { Leipzig, Telefon } 0341678-0 \text {, info@leipziger- } \\
\text { messe.de, www.leipziger-buchmesse.de }\end{array}$ \\
\hline $\begin{array}{l}\text { 27. bis 28. März } \\
\text { Trier }\end{array}$ & $\begin{array}{l}\text { Forschung + Datenmanagement }= \\
\text { Forschungsdatenmanagement - Wann geht die } \\
\text { Gleichung auf? } \\
\text { Implementierung einer institutionellen FDM- } \\
\text { Infrastruktur- und -Service-Landschaft }\end{array}$ & $\begin{array}{l}\text { Dr. André Förster, Universität Trier, Servicezentrum eSciences, } \\
\text { Universitätsring, DM-Gebäude, Raum 145, } 54286 \text { Trier, } \\
\text { Telefon } 0651201 \text { - 2104, foerster@uni-trier.de, www. } \\
\text { esciences.uni-trier.de/events/event/forschung-datenmana- } \\
\text { gement-forschungsdatenmanagement-wann-geht-die-glei- } \\
\text { chung-auf-implementierung-einer-institutionellen-fdm-infra- } \\
\text { struktur-und-\%e2 \%80\%91service-landschaft/ }\end{array}$ \\
\hline $\begin{array}{l}\text { 1. bis 5. April } \\
\text { Hannover }\end{array}$ & Hannover Messe & $\begin{array}{l}\text { Deutsche Messe, Messegelände, } 30521 \text { Hannover, Telefon } \\
0511 \text { 89-0, Fax } 0511 \text { 89-32626, www.hannovermesse.de }\end{array}$ \\
\hline
\end{tabular}




\begin{tabular}{ll}
\hline $\begin{array}{l}\text { 8. bis 10. April } \\
\text { Leipzig }\end{array}$ & $\begin{array}{l}\text { vfm Frühjahrstagung } \\
\text { Medien, Mining, Metadaten } \\
\text { Visionen für die Mediendokumentation }\end{array}$ \\
\hline $\begin{array}{l}\text { 9. bis 10. April } \\
\text { Münster }\end{array}$ & 59. PAID-Tagung \\
\hline $\begin{array}{l}\text { 10. bis 11. April } \\
\text { Berlin }\end{array}$ & Big-Data.Al Summit 2019 \\
\hline $\begin{array}{l}\text { 14. April } \\
\text { Köln }\end{array}$ & $\begin{array}{l}8^{\text {th }} \text { international workshop on Bibliometric- } \\
\text { enhanced Information Retrieval (BIR 2019) }\end{array}$
\end{tabular}

vfm - Verein für Medieninformation und

Mediendokumentation, Prof. Dr. Ralph Schmidt, rais.schmidt@t-online.de,www.vfm-online.de/tagungen/

PAID Pharma Arbeitskreis Information Dokumentation, Susanne Rehm, c/o DHU-Arzneimittel GmbH \& Co.KG, Ottostraße 24,76227 Karlsruhe, susanne.rehm@dhu.de, paid.de/neue-seite/wordpress/naechste-tagung/

Bitkom e.V. und Bitkom Servicegesellschaft $\mathrm{mbH}$, Albrechtstraße 10, 10117 Berlin, www.big-data.ai/de GESIS-Leibniz-Institut für Sozialwissenschaften, Unter Sachsenhausen 6-8, 50667 Köln, www.gesis.org/en/ services/events/events-archive/conferences/ecir-workshops/ecir-workshop-2019

\begin{tabular}{ll}
\hline 14. bis 18. April & $41^{\text {st }}$ European Conference on Information \\
Köln & Retrieval
\end{tabular}

GESIS-Leibniz-Institut für Sozialwissenschaften, Unter Sachsenhausen 6-8, 50667 Köln, Telefon 49(0)221/47694598, http://ecir2019.org

\begin{tabular}{ll}
\hline 25. April & DTT-Vertiefungsseminar \\
Köln & Terminologiearbeit -Projekte, Prozesse, \\
& Datenaustausch
\end{tabular}
Deutscher Terminologie-Tag e.V. (DTT), Geschäftsstelle, c/o Technische Hochschule Köln, Institut für Informationsmanagement (IIM), Claudiusstraße 1, 50768 Köln, geschaeftsstelle@dttev.org, www. http://dttev.org/

\begin{tabular}{ll}
\hline $\begin{array}{l}\text { 26. April } \\
\text { Hamburg }\end{array}$ & $\begin{array}{l}\text { Thementag Digitalisierung im } \\
\text { Gesundheitswesen }\end{array}$ \\
\hline $\begin{array}{l}\text { 6. bis 10. Mai } \\
\text { Frankfurt am Main }\end{array}$ & DGl-Zertifikatslehrgang Informationsassistenz
\end{tabular}

AKI RP/Eifel, Werner Povoden, Adolf Meier Straße 68, 32758 Detmold, Telefon 05231308 1374, info@cspcampus.de

DGI-Geschäftsstelle, Windmühlstraße 3, 60329 Frankfurt am Main, Telefon 069 430313, Fax 069 4909096, informationsassistent@dgi-info.de,www.dgi-info.de

\begin{tabular}{ll}
\hline $\begin{array}{l}\text { 8. Mai } \\
\text { Frankfurt am Main }\end{array}$ & $\begin{array}{l}\text { DGI-Praxisseminar Kommunikation für } \\
\text { Information Professionals }\end{array}$ \\
\hline $\begin{array}{l}\text { 8. Mai } \\
\text { Dresden }\end{array}$ & $\begin{array}{l}\text { Frühjahrstreffen der Fachgruppe Dokumentation } \\
\text { im Deutschen Museumsbund }\end{array}$
\end{tabular}

DGI-Geschäftsstelle, Windmühlstraße 3, 60329 Frankfurt am Main, Telefon 069 430313, Fax 069 4909096, informationsassistent@dgi-info.de,www.dgi-info.de

Frank von Hagel, Institut für Museumsforschung, In der Halde 1, 14195 Berlin, Telefon: (030) 8301-460, Fax: (030) 8301-504, E-Mail: f.v.hagel@smb.spk-berlin.de, www.museumsbund.de

\begin{tabular}{ll}
\hline $\begin{array}{l}\text { 10. Mai } \\
\text { Frankfurt am Main }\end{array}$ & DGI-Workshop Urheberrecht \\
\hline $\begin{array}{l}\text { 10. Mai } \\
\text { Frankfurt am Main }\end{array}$ & $\begin{array}{l}\text { ILO Conference on Learning Information Literacy } \\
\text { across the Globe }\end{array}$ \\
\hline $\begin{array}{l}\text { 13. bis 14. Mai } \\
\text { Düsseldorf }\end{array}$ & $\begin{array}{l}\text { Mai-Treffen der Fachgruppe Dokumentation im } \\
\text { Deutschen Museumsbund }\end{array}$
\end{tabular}
DGI-Geschäftsstelle, Windmühlstraße 3, 60329 Frankfurt am Main, Telefon 069 430313, Fax 069 4909096, informationsassistent@dgi-info.de,www.dgi-info.de

DIPF | Leibniz-Institut für Bildungsforschung und Bildungsinformation, Rostocker Straße 6, 60323 Frankfurt am Main, https://informationliteracy.eu/conference

Frank von Hagel, Institut für Museumsforschung, In der Halde 1, 14195 Berlin, Telefon: (030) 8301-460, Fax: (030) 8301-504, E-Mail: f.v.hagel@smb.spk-berlin.de, www.museumsbund.de

\begin{tabular}{ll}
\hline $\begin{array}{l}\text { 14. bis 15. Mai } \\
\text { Frankfurt am Main }\end{array}$ & $\begin{array}{l}\text { DGI-Praxisseminar Recherchewerkzeug So } \\
\text { Media gekonnt einsetzen }\end{array}$ \\
\hline $\begin{array}{l}\text { 13. bis 17. Mai } \\
\text { Frankfurt am Main }\end{array}$ & DGI-Zertifikatslehrgang Informationsassis \\
\hline $\begin{array}{l}\text { 21. Mai } \\
\text { Mainz }\end{array}$ & $\begin{array}{l}\text { Tagung der DGI-Fachgruppe Akademische } \\
\text { Integrität }\end{array}$
\end{tabular}
DGI-Geschäftsstelle, Windmühlstraße 3, 60329 Frankfurt am Main, Telefon 069 430313, Fax 0694909096 , informationsassistent@dgi-info.de,www.dgi-info.de DGI-Geschäftsstelle, Windmühlstraße 3, 60329 Frankfurt am Main, Telefon 069 430313, Fax 069 4909096, informationsassistent@dgi-info.de,www.dgi-info.de Dominik Schuh, Universitätsbibliothek Mainz, Jakob-WelderWeg 6, 55128 Mainz, Telefon 06131-39-23370, dschuh@unimainz.de, www.akin.uni-mainz.de, www.dgi-info.de/ akademische-integritaet/tagung2019

\begin{tabular}{ll}
\hline 4. bis 6. Juni & WissKom2019- Forschungsdaten: Sammeln, \\
Jülich & sichern, strukturieren
\end{tabular}

Forschungszentrum Jülich, Zentralbibliothek, 52425 Jülich, Telefon 0246161-2907, zb-konferenz@fz-juelich.de, www.wisskom2019.de 


\begin{tabular}{|c|c|c|}
\hline $\begin{array}{l}\text { 5. bis } 7 . \text { Juni } \\
\text { Ilmenau }\end{array}$ & $\begin{array}{l}\text { PATINFO } 2019 \\
\text { 41. Kolloquium der Technischen Universität } \\
\text { Ilmenau über Patentinformation }\end{array}$ & $\begin{array}{l}\text { Astrid Schieck, Technische Universität Ilmenau, PATON, } \\
\text { Landespatentzentrum Thüringen, Postfach } 1005 \text { 65, } 98684 \\
\text { Ilmenau, Telefon } 03677 \text { 69-4572, Fax } 03677 \text { 69-4538, } \\
\text { patinfo@tu-ilmenau.de, www.paton.tu-ilmenau.de }\end{array}$ \\
\hline $\begin{array}{l}\text { 8. bis 12. Juli } \\
\text { Wien, Österreich }\end{array}$ & $\begin{array}{l}\text { TSS } 2019 \text { - The International Terminology } \\
\text { Summer School }\end{array}$ & $\begin{array}{l}\text { TermNet Business GmbH, Gabriele Sauberer, } \\
\text { Mooslackengasse 17,1190 Wien, Österreich, } \\
\text { info@termnet-gmbh.at, www.termnet.eu }\end{array}$ \\
\hline $\begin{array}{l}\text { 29. Juni bis 2. Juli } \\
\text { Frankfurt am Main }\end{array}$ & Tendence & $\begin{array}{l}\text { Messe Frankfurt Exhibition GmbH, Ludwig-Erhard-Anlage 1, } \\
60327 \text { Frankfurt am Main, Telefon 069 7575-6866, } \\
\text { tendence@messefrankfurt.com, www.tendence.com }\end{array}$ \\
\hline $\begin{array}{l}\text { 20. August bis } \\
\text { 1. September } \\
\text { Montreux, Schweiz }\end{array}$ & Schweizer Bibliothekskongress 2019 & $\begin{array}{l}\text { BIS, Bleichemattstrasse 42, } 5000 \text { Aarau, Schweiz, } \\
\text { Telefon }+41628231938 \text {, Fax +41 } 6282319 \text { 39, info@bis.ch }\end{array}$ \\
\hline $\begin{array}{l}\text { 10. bis 13. September } \\
\text { Graz, Österreich }\end{array}$ & $\begin{array}{l}\text { 34. Österreichischer Bibliothekartag } \\
\text { Künstliche Intelligenz und Bibliotheken }\end{array}$ & $\begin{array}{l}\text { vÖB, Telefon +43-1-4277-17677, bibliothekartag2019@voeb- } \\
\text { b.at, bibliothekartag2019.voeb-b.at }\end{array}$ \\
\hline $\begin{array}{l}\text { 16. bis 18. September } \\
\text { Lutherstadt Wittenberg }\end{array}$ & $\begin{array}{l}\text { 4. DGI-Forum Wittenberg } \\
\text { KI macht Schule }\end{array}$ & $\begin{array}{l}\text { DGI-Geschäftsstelle, Windmühlstraße 3, } 60329 \text { Frankfurt am } \\
\text { Main, Telefon } 069 \text { 430313, Fax } 069 \text { 4909096, } \\
\text { wittenberg2019@dgi-info.de, www.dgi-info.de }\end{array}$ \\
\hline $\begin{array}{l}\text { 17. bis 20. September } \\
\text { Suhl }\end{array}$ & $\begin{array}{l}\text { 89. Deutscher Archivtag } \\
\text { RECHTsicher - Archive und ihr rechtlicher } \\
\text { Rahmen }\end{array}$ & $\begin{array}{l}\text { DGI-Geschäftsstelle, Windmühlstraße 3, } 60329 \text { Frankfurt am } \\
\text { Main, Telefon } 069 \text { 430313, Fax } 069 \text { 4909096, } \\
\text { wittenberg2019@dgi-info.de, www.dgi-info.de }\end{array}$ \\
\hline $\begin{array}{l}\text { 8. bis 9. Oktober } \\
\text { Osnabrück }\end{array}$ & $\begin{array}{l}\text { Jahrestagung der Deutschen Initiative für } \\
\text { Netzwerkinformation (DINI) }\end{array}$ & $\begin{array}{l}\text { DINI-Geschäftsstelle, Claudia Walther c/o Niedersächsische } \\
\text { SUB Göttingen, Platz der Göttinger Sieben 1, } 37073 \text { Göttingen, } \\
\text { Telefon: } 0551 \text { 39-33857, gs@dini.de, https://dini.de/nachrich } \\
\text { ten/nachricht/news/20-dini-jahrestagung-2019 }\end{array}$ \\
\hline $\begin{array}{l}\text { 16. bis 20. Oktober } \\
\text { Frankfurt am Main }\end{array}$ & $\begin{array}{l}\text { 71. Frankfurter Buchmesse } \\
\text { Ehrengast Norwegen }\end{array}$ & $\begin{array}{l}\text { Ausstellungs- und Messe GmbH, Frankfurter Buchmesse, } \\
\text { Braubachstraße 16, 60311 Frankfurt am Main, Telefon } 069 \\
\text { 2102-0, Fax } 069 \text { 2102-227/-277, info@book-fair.com, } \\
\text { www.buchmesse.de }\end{array}$ \\
\hline $\begin{array}{l}\text { 19. bis 23. Oktober } \\
\text { Melbourne, Australien }\end{array}$ & $82^{\text {nd }}$ ASIS\&T Annual Meeting & $\begin{array}{l}\text { ASIS\&T Headquarters, } 8555 \text { 16th Street, Suite 850, Silver } \\
\text { Spring, Maryland 20910, USA, Telefon } 301495 \text { 0900, } \\
\text { asist@asist.org }\end{array}$ \\
\hline $\begin{array}{l}\text { 21. bis 23. Oktober } \\
\text { Berlin }\end{array}$ & $\begin{array}{l}\text { Berliner Herbsttreffen der } \\
\text { Museumsdokumentation }\end{array}$ & $\begin{array}{l}\text { Frank von Hagel, Institut für Museumsforschung, } \\
\text { In der Halde 1, 14195 Berlin, Telefon: (030) 8301-460, Fax: } \\
\text { (030) 8301-504, E-Mail: f.v.hagel@smb.spk-berlin.de, } \\
\text { www.museumsbund.de }\end{array}$ \\
\hline $\begin{array}{l}\text { 12. bis 13. November } \\
\text { Stuttgart }\end{array}$ & $\begin{array}{l}\text { 15. Stuttgarter Wissensmanagement-Tage } \\
\text { „Wissen verbindet“ }\end{array}$ & $\begin{array}{l}\text { Oliver Lehnert, wissensmangement - Das Magazin für } \\
\text { Führungskräfte, Telefon } 0821486 \text { 852-92, } \\
\text { Telefax:0821 } 486 \text { 852-93, service@wissensmanagement.net, } \\
\text { www.wissensmanagement.net }\end{array}$ \\
\hline $\begin{array}{l}\text { 12. bis 14. November } \\
\text { Stuttgart }\end{array}$ & tekom-Jahrestagung 2019 & $\begin{array}{l}\text { Gesellschaft für technische Kommunkiation - tekom } \\
\text { Deutschland e.V., Rotebühlstraße 64, } 70178 \text { Stuttgart, } \\
\text { Telefon } 071165704-0 \text {, info@tekom.de, www.tekom.de }\end{array}$ \\
\hline $\begin{array}{l}\text { 27. bis 29. November } \\
\text { Berlin }\end{array}$ & OEB 2019 & $\begin{array}{l}\text { Rebecca Stromeyer, ICWE GmbH, Leibnizstraße 32, } 10625 \\
\text { Berlin, Telefon } 030 \text { 3101818-0, info@online-educa.com, } \\
\text { www.online-educa.com }\end{array}$ \\
\hline $\begin{array}{l}\text { 3. Dezember } \\
\text { Mannheim }\end{array}$ & BibliotheksLeiterTag 2019 & OCLC, www.bibliotheksleitertag.de \\
\hline
\end{tabular}


